\title{
Relation Between Chronic Sinusitis And Deviated Nasal Septum
}

\author{
Dr. Mohd Ajmal*,Dr Nema Usman* \\ *Department Of Anatomy JNMC AMU Aligarh
}

\section{Introduction}

Sinusitis is one of the most common diseases of the nose and paranasal sinuses. It is the fifth most common diagnosis for which antibiotics are prescribed [1]. Chronic sinusitis (CS) is an extremely prevalent disorder affecting up to two percent of the world population, has a significant impact on the quality of life of affected individual [2].

The term 'sinusitis' refers to a group of disorders characterized by inflammation of mucosa of nose and paranasal sinuses. Deviated Nasal septum is a common disorder that presents up to $62 \%$ of the population, and its role in the pathogenesis of chronic sinusitis remains uncertain [3]. Hippocrates in $5^{\text {th }}$ century B.C stated that "In a person having a painful spot in head, with intense headaches, pus or fluid running from the nose removes the disease", which may be referred to as describing sinusitis [4].Anatomically, areas of mucosal contacts are most likely to occur in the narrow mucosal lined channels of the middle meatus and ethmoid air cell system [5].

\section{Objective Of The Study}

To study the association and relationship of chronic sinusitis with deviated nasal septum with

\section{Material And Methods}

This study was conducted in department of ENT, JNMC AMU Aligarh

All the patients with symptomtomatic sinusitis of more than 12 weeks duration are included in the study.

Sample size: 150 patients

Patients age $>15$ years and $\leq 60$ years.

\section{TFR Diagnostic criteria for sinusitis}

Inclusive criteria : The presence of two or more major factors, or one major and two minor factors, is considered suggestive of sinusitis.

\section{Methods of collection of data:}

The cases selected for the study were subjected to

1. Clinical examination.

2. Diagnostic nasal endoscopy:

3. X-ray of nose and paranasal sinuses

4. CT scan of nose and paranasal sinuses (in required cases)

After clinical examination patients with suspected sinusitis were subjected to radiological investigations.

\section{Results}

Table 1. Showing sex distribution

\begin{tabular}{|l|l|l|}
\hline Gender & No. of patients & $\%$ \\
\hline MALE & 88 & 58.67 \\
\hline FEMALE & 62 & 41.33 \\
\hline TOTAL & 150 & 100 \\
\hline
\end{tabular}

12 patients were in the age group of $\leq 20 \mathrm{yrs}(8 \%)$,

48 of them in age group of 21-30 years (32\%) and

39 patients in age group of $31-40$ years $(26 \%)$,

36 patients were in $41-50$ years $(24 \%)$ and

15 patients in $51-60$ years $(10 \%)$. 
Youngest patient was 17 years and oldest patient was 58 years

Table 2

\begin{tabular}{|c|c|c|c|}
\hline GENDER & $\begin{array}{l}\text { Number of Patient with } \\
\text { Sinusitis }\end{array}$ & $\begin{array}{l}\text { Number sinusit } \\
\text { Patient with DNS }\end{array}$ & $\%$ \\
\hline MALE & 88 & 73 & 83 \\
\hline FEMALE & 62 & 55 & 88.7 \\
\hline TOTAL & 150 & 128 & \\
\hline
\end{tabular}

Table 3

\begin{tabular}{|l|l|l|l|}
\hline Age group & Pt without DNS & Pt with DNS & \% of pt with DNS \\
\hline $15-20$ YEAR & 12 & 10 & $83.33 \%$ \\
\hline $21-30$ YEAR & 48 & 45 & $93.75 \%$ \\
\hline $31-40$ YEAR & 39 & 33 & $84.60 \%$ \\
\hline $41-50$ YEAR & 36 & 31 & $86.11 \%$ \\
\hline $51-60$ YEAR & 15 & 9 & $60 \%$ \\
\hline
\end{tabular}

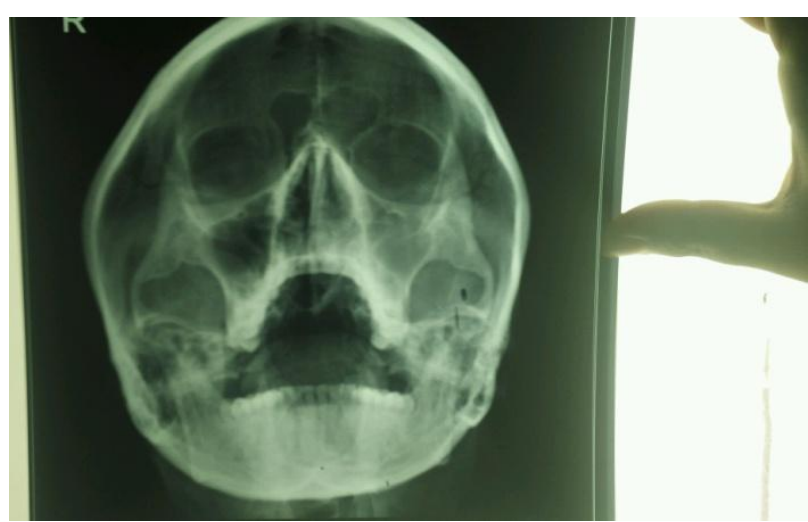

fig 1 showing left maxillary sinusitis with DNS

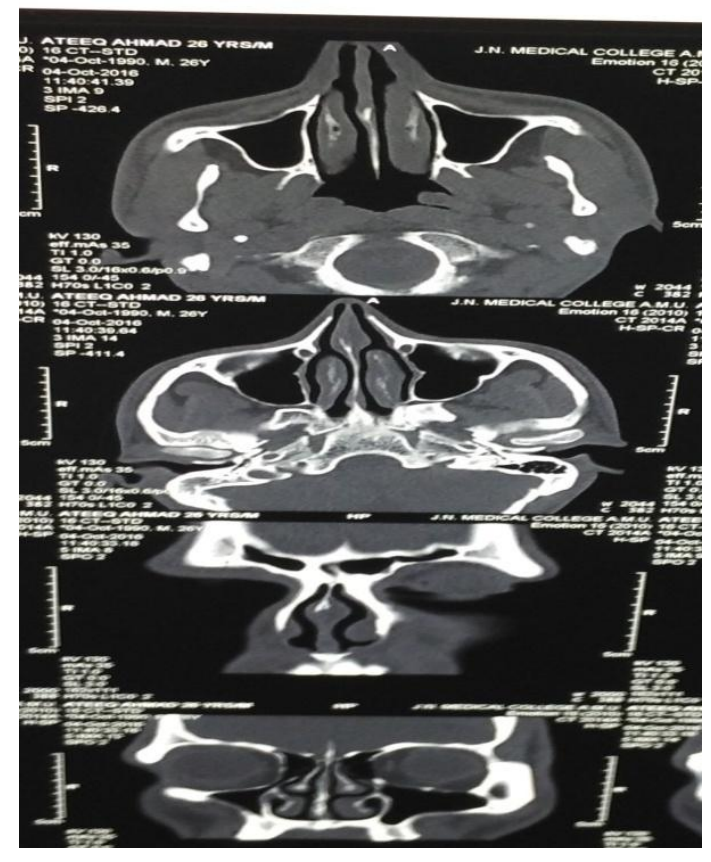




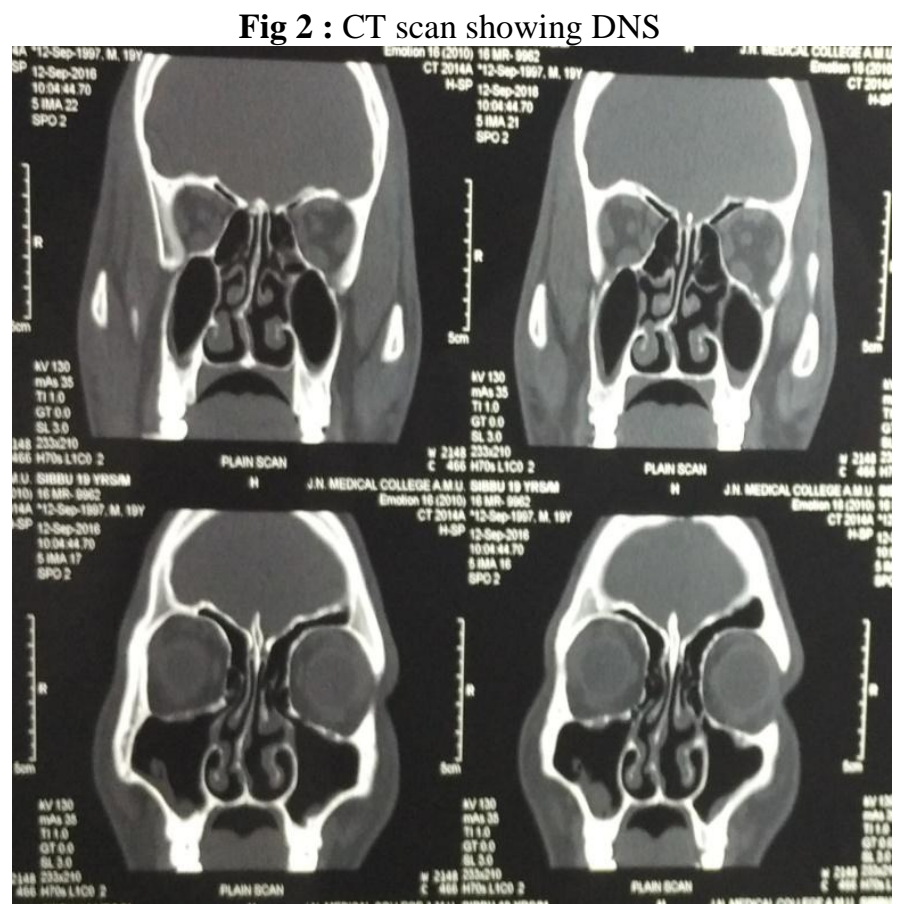

Fig 3 : Ct Scan Depicting Dns

Total of 150 patients were enrolled in the study between 15 and 60 years old.

All the patients presenting with symptoms of sinusitis of more than 12 weeks duration with 2 major and one minor or 2 minor symptoms were included in the study. Patients diagnosed as cases of sinusitis based on TFR criteria [14]. Out of 150 patients with diagnosed sinusitis 128 also displayed DNS.

\section{Discussion}

A total of 150 patients were enrolled in the study between 15 and 60 years old. All the patients presenting with symptoms of sinusitis of more than 12 weeks.Patients diagnosed as cases of rhinosinusitis based on TFR criteria [14]. There are three theories explaining pathophysiological relation between the deviated nasal septal and chronic sinusitis. The first of these is the mechanical theory which states that secretions accumulates in the sinus as a result of narrowing of the ostiomeatal complex and thus infections ensues in the retained secretions and causes chronic rhinosinusitis. The second theory is the aerodynamic theory. According to this theory, the mucociliary activity decreases following the nasal flow rate increase and mucosal dryness in relation with the nasal septal deviation and consequently, chronic rhinosinusitis develops.

The third theory is the Bachert's pressure theory. According to this theory, deviation of the posterior nasal septum causes chronic rhinosinusitis by creating pressure and air flow changes within the maxillary sinuses $[10,11]$. In the present study the incidence of DNS was more in female than male with $88.7 \%$ female and $83 \%$ male. In a study by Madani et al, there were $68.3 \%$ male and $31.7 \%$ female with [12]. Ozkurt et al in his study observed that incidence was more in male as compared to female [13]. In our study most patients had bilateral maxillary sinusitis in 72 patients $(48 \%)$ while unilateral maxillary sinusitis was seen in 60 patients (40 $\%$ ), bilateral frontal sinusitis was seen in 52 patients $(34.67 \%)$, while unilateral frontal sinusitis was seen in 18 patients $(12 \%)$, bilateral ethmoidal sinusitis was presented in 40 patients $(26.67 \%)$ while unilateral ethmoidal sinusitis was seen in 19 patients $(12.6 \%)$ and bilateral sphenoidal sinusitis was seen in 9 patients $(6 \%) .9$ patients had pansinusitis $(6 \%)$ while no patient presented with unilateral sphenoidal sinusitis.

In a study by Mohebbi et al, bilateral maxillary sinusitis presentation was seen in $27 \%$ of patients while unilateral presentation was seen in $18.4 \%$, similarly unilateral frontal sinus involvement was seen in $12.5 \%$ and bilaterally in $11.2 \%$, bilateral ethmoidal sinusitis was seen in $36.1 \%$ and unilateral ethmoidal sinusitis was seen in $18.1 \%$, bilateral sphenoidal sinusitis was seen in $12.3 \%$, unilateral sphenoid sinusitis was seen in $13 \%$ [9]. Rao et al in their study found horizontal spur was most commonly associated in sinus pathology, $43.4 \%$ of patients spurs were accounted for sinus pathology, similarly type III (posterior vertical deviation) was seen having osteo meatal complex (OMC) block in $62.5 \%$ of cases, S-shaped deviation was associated with OMC block in $50 \%$ of cases [8].In this study we found that the prevalence of nasal septal deviations and the sinusitis was significant [p-value is $<0.001]$. 


\section{Conclusion}

Present study showed that chronic sinusitis was almost equally prevalent in males and female. Headache was the most common symptom followed by nasal blockage due to pressure and airflow changes caused by DNS. C-shaped deviation was the most common presentation, being more common on the left side.Bilateral Maxillary sinusitis was the commonest presentation, more so in association with C-shaped DNS. Pansinusitis was only associated with S-shaped deviation, since S-shaped DNS obstructs the laminar airflow pattern in both the nasal cavities.Isolated sphenoid sinusitis was not seen as the sinuses occupy a midline position. The ostium occupies a medial and superior position on either side of the nasal septum; hence obstruction of the ostia due to DNS is less commonly

\section{Refrences}

[1]. Rosenfeld RM, Piccirillo JF, Chandrasekhar SS, Itzhak Brook I, Kumar KA, Kramper M, et al. Clinical Practice Guideline (Update): Adult Sinusitis. Otolaryngology-Head and Neck Surgery 2015, Vol. 152(2S) S1-S39.

[2]. Rodney J Schlosser and David W Kennedy, Nasal endoscopy, Scott-Brawn's Otorhinolaryngology, Head and Neck Surgery, volume 2:2008, pg 1344 .

[3]. Lloyd G. CT of the paranasal sinuses: study of a control series in relation to endoscopic sinus surgery. J LaryngolOtol. 1990; 104:477-481.

[4]. Lusk RP, McAlister B, Ahmed F. Anatomic variations in Pediatric chronic sinusitis. A CT study. OCNA 1996; $29: 75-91$.

[5]. Kennedy DW, Zinreich J, Arthur E, Rosenbaum, Johns ME. Theory and diagnostic evaluation. Arch Otolaryngol Head and Neck Surg 1985; 111(9): 576- 582 .

[6]. Collet S, Bertrand B, Cornu S, Eloy P, Rombaux P. Is septal deviation a risk factor for chronic sinusitis? Review of literature. Acta Otorhinolaryngol Belg.2001; 55(4):299-304.

[7]. Rao JJ, Kumar ECV, et al. Classification of nasal septal deviations-relation to sinonasal pathology," Indian journal of otolaryngology and head and neck surgery 2005; 57(3): 199-201.

[8]. Moorthy PNS, Kolloju S, Madhira S, Jowka AB. Clinical study on deviated nasal septum and its associated pathology. International Journal of Otolaryngology and Head \& Neck Surgery, 2014, 3, 75-81.

[9]. Mohebbi et al.: An epidemiologic study of factors associated with nasal septum deviation by computed tomography scan: a cross sectional study. BMC Ear, Nose and Throat Disorders 2012 12:15

[10]. Brown S. Anatomy of nose and paranasal sinuses. In: Lund VJ, H Stammberger, Scott Brown Otolaryngology, Basic Sciences; 5, Butterworth-Heinemann; oxford, $7^{\text {th }}$ edn; 2008: Pg1318.

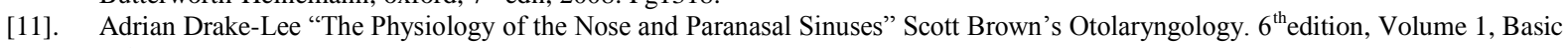
Sciences pp 1/6/11-15.

[12]. Madani SA et al: Association between anatomical variations of the sinonasal region and chronic rhino sinusitis: A Prospective Case Series Study. Scientific Journal of the Faculty of Medicine in Niš 2013; 30(2): 73-77.

[13]. Ozkurt FE et al., Relation Between the Nasal Septal Deviation and Chronic Rhinosinusitis, International Journal of Basic and Clinical Studies (IJBCS) 2014; 3(1): 25-30. 